\title{
WEAKLY CONTINUOUS NONLINEAR ACCRETIVE OPERATORS IN REFLEXIVE BANACH SPACES
}

\author{
W. E. FITZGIBBON
}

Abstract. Let $X$ be a reflexive space and $A$ be a weakly continuous (possibly nonlinear) operator which maps $X$ to $X$. We are concerned with the autonomous differential equation

$$
u^{\prime}(t)+A u(t)=0, \quad u(0)=X .
$$

We first provide a local solution to (1.1) and then we apply the additional hypothesis that $A$ is accretive to extend the local solution of (1.1) to a global solution. If $A$ is weakly continuous and accretive then $A$ is shown to be $m$-accretive, i.e. $R(I+\lambda \cdot A)=X$ for all $i \geqq 0$. The $m$-accretiveness of $A$ enables us to provide a semigroup representation of solutions to (1.1),

$$
u(t)=T(t) x=\lim _{n \rightarrow \infty}(I+(t / n) A) x{ }^{n} \text { for } t \in[0, \infty) .
$$

We then investigate properties of semigroups which have weakly continuous infinitesimal generators.

1. Introduction and definitions. The subject of this paper is the autonomous differential equation

$$
u^{\prime}(t)+A u(t)=0, \quad u(0)=x,
$$

where $A$ is a nonlinear weakly continuous accretive operator mapping a reflexive Banach space into itself. We solve (1.1) locally, extend the solution, and then investigate properties of the semigroup associated with it. In recent articles $T$. Kato [5] has considered equations of form (1.1) where $A$ is a demicontinuous accretive operator on a Banach space which has uniformly convex dual; R. H. Martin [6] has considered the case of $A$ being continuous and accretive on a general Banach space; and $\mathrm{S}$. Chow and J. D. Schuur [2] provide a local solution to (1.1) in case $A$ is weakly continuous on a separable, reflexive Banach space.

Presented to the Society, January 20, 1972 under the title Weakly continuous accretive operators in reflexive Banach spaces; received by the editors November 9, 1972 and, in revised form, March 8, 1973.

AMS (MOS) subject classifications (1970). Primary 47H15, 34605; Secondary 47B44, 47D05.

Key words and phrases. Accretive, weakly continuous, semigroup of nonexpansive nonlinear operators. 
Definition 1.1. An operator $A$ is said to be accretive provided that $\|(x+\lambda A x)-(y+\lambda A y)\| \geqq\|x-y\|$ for all $x, y \in D(A)$ and $\lambda \geqq 0$. T. Kato [5] has shown that this definition is equivalent to the statement that $\operatorname{Re}(A x-A y, f) \geqq 0$ for some $f \in F(x-y)$ where $F$ is the duality map from $X$ to $X^{*}$.

Definition 1.2. An accretive operator $A$ is said to be an $m$-accretive operator provided $R(I+\lambda A)=X$ for all $\lambda \geqq 0$. It is well known that an $m$-accretive operator is a maximal accretive operator. However, not every accretive operator can be extended to an $m$-accretive operator.

Definition 1.3. A function $u:[0, T) \rightarrow X$ is said to be a strong solution to the Cauchy problem

$$
d u(t) / d t+A u(t)=0, \quad u(0)=a,
$$

provided that $u$ is Lipschitz continuous on each compact subset of $[0, T)$, $u(0)=a, u$ is strongly differentiable almost everywhere and $d u(t) / d t+$ $A u(t)=0$ for a.e. $t \in[0, T)$.

DEFINITION 1.4. An operator is said to be weakly continuous if it maps weakly convergent sequences to weakly convergent sequences.

2. An existence theorem and semigroup representation. The first lemma provides a local solution to (1.2).

Lemma 2.1. Let $X$ be a reflexive Banach space and suppose that $A$ is a weakly continuous operator with $D(A)=X$. Then there is a finite interval $[0, T)$ such that the Cauchy problem (1.2) has a strong solution no $[0, T)$.

Proof. Since $A$ is weakly continuous, it is locally bounded. Thus, if $a \in X$ there exist $r, K>0$ such that if $x \in S_{r}(a)$ then $\|A x\| \leqq K$. Let $\varepsilon_{n} \downarrow 0$. We shall recursively define a sequence of functions which solve the approximate equations,

$$
\begin{array}{ll}
d u_{n}(t) / d t+A u_{n}\left(t-\varepsilon_{n}\right)=0, \quad u_{n}(0)=a . \\
u_{n}(t)=a & \text { if } t<0 . \\
=a-\int_{0}^{t} A u_{n}\left(s-\varepsilon_{n}\right) d s & \text { if } t \in\left[j \varepsilon_{n},(j+1) \varepsilon_{n}\right], \\
& j=0,1, \cdots,\left[r / K / \varepsilon_{n}\right]-1 .
\end{array}
$$

We wish to argue that $u_{n}(t) \in S_{r}(a)$. Clearly if $t \in\left[0, \varepsilon_{n}\right]$ then $\left\|u_{n}(t)-a\right\|=$ $\|t A a\| \leqq r$. Assuming the desired result for $t \in\left[0, j \varepsilon_{n}\right]$ consider $t \in$ $\left[0,(j+1) \varepsilon_{n}\right]$. Then

$$
\begin{aligned}
\left\|u_{n}(t)-a\right\| & \leqq\left\|\int_{0}^{t} A u_{n}\left(s-\varepsilon_{n}\right) d s\right\| \\
& \leqq t \max _{s \in\left[0, j \varepsilon_{n}\right]}\left\|A u_{n}(s)\right\| \leqq r / K(K)=r .
\end{aligned}
$$


Thus $u_{n}(t) \in S_{r}(a)$. By observing that

$$
\left\|u_{n}(t)-u_{n}(\tau)\right\| \leqq\left\|\int_{\tau}^{t} A u_{n}\left(s-\varepsilon_{n}\right) d s\right\| \leqq|t-\tau| K,
$$

we see that the sequence $\left\{u_{n}(t)\right\}$ is uniformly Lipschitz continuous in $t$.

Let $E$ be an enumeration of a countable dense subset of $[0, r / K]$. By employing a diagonalization we obtain a subsequence $\left\{u_{n^{\prime}}(t)\right\}$ such that $u_{n},\left(t_{i}\right)$ converges weakly for each $t_{i} \in E$. We relabel this sequence $\left\{u_{n}(t)\right\}$. To show that $u_{n}(t)$ converges weakly for $t \in[0, r / K]$ we observe that if $f \in X^{*}$

$$
\begin{aligned}
\left|\left(u_{n}(t)-u_{m}(t), f\right)\right| \leqq & \left|\left(u_{n}(t)-u_{n}\left(t_{k}\right), f\right)\right|+\left|\left(u_{n}\left(t_{k}\right)-u_{m}\left(t_{k}\right), f\right)\right| \\
& +\left|\left(u_{m}(t)-u_{m}\left(t_{k}\right), f\right)\right| .
\end{aligned}
$$

Thus by choosing $t_{k} \in E$ so that $2\left|t-t_{k}\right| K\|f\|<2 \varepsilon / 3$ and by choosing $N>0$ so that $n, m>N$ imply $\left|\left(u_{n}\left(t_{k}\right)-u_{m}\left(t_{k}\right), f\right)\right|<\varepsilon / 3$ we insure that $\left|\left(u_{n}(t)-u_{m}(t), f\right)\right|<\varepsilon$. Since reflexive spaces are weakly complete, there is a $u(t)=w-\lim u_{n}(t)$.

We shall now show that $u(t)$ is Lipschitz continuous. If $f \in X^{*}, t$, $\tau \in[0, r / K), \lim \left(u_{n}(t)-u_{n}(\tau), f\right)=(u(t)-u(\tau), f)$. Thus if $\varepsilon$ is any positive number and $f \in F(u(t)-u(\tau))\left(F\right.$ is the duality map from $X$ to $\left.X^{*}\right)$, there is an $N>0$ such that $n>N$ implies

$$
\left(u_{n}(t)-u_{n}(\tau), f\right)+\varepsilon>(u(t)-u(\tau), f)=\|u(t)-u(\tau)\|^{2} .
$$

Thus for any $\varepsilon>0$

$$
\|u(t)-u(\tau)\|^{2}<\varepsilon+\left|\left(u_{n}(t)-u_{n}(\tau), f\right)\right| \leqq \varepsilon+|t-\tau| K\|u(t)-u(\tau)\|
$$

and hence $\|u(t)-u(\tau)\| \leqq|t-\tau| K$.

Clearly $u_{n}\left(t-\varepsilon_{n}\right) \longrightarrow u(t)$ and hence $A u_{n}\left(t-\varepsilon_{n}\right) \rightarrow A u(t)$. If $f \in X^{*}$ by taking limits of the equation $\left(u_{n}(t), f\right)=(a, f)-\int_{0}^{t}\left(A u_{n}\left(s-\varepsilon_{n}\right), f\right) d s$ we obtain

$$
(u(t), f)=(a, f)-\int_{0}^{t}(A u(s), f) d s, \quad t \in[0, r / K) .
$$

Applying standard techniques to (2.2) we see that $u(t)=a-\int_{0}^{t} A u(s) d s$, $t \in[0, r / K)$, and hence that

$$
u^{\prime}(t)+A u(t)=0 \text { for a.e. } t \in[0, r / K) .
$$

In the next theorem we shall see that if we also require that the operator $A$ is accretive then the local solution of the preceding lemma may be extended to a global solution. 
THEOREM 2.1. Let $X$ be a reflexive Banach space and suppose that $A$ is a weakly continuous accretive operator with $D(A)=X$. Then the Cauchy problem (1.2) has a unique strong solution on $[0, \infty)$.

Proof. From the previous lemma it is clear that there exists a local solution to (1.4) on a maximum interval existence $[0, T)$. We wish to show that $T<\infty$ leads to a contradiction. Let $t, t+h \in[0, T)$, then by a lemma of $T$. Kato [5].

$$
(d / d t)\left(\|u(t+h)-u(t)\|^{2}\right)=-2 \operatorname{Re}\langle A u(t+h)-A u(t), f\rangle
$$

where $f \in F(u(t+h)-u(t))$. By the accretiveness of $A$, we see that

$$
-2 \operatorname{Re}\langle A u(t+h)-A u(t), f\rangle \leqq 0
$$

Thus, $\|u(t+h)-u(t)\| \leqq\|u(h)-a\|$ and hence $\|A u(t)\| \leqq\|A a\|$. If $t$, $\tau \in[0, T)$,

$$
\|u(t)-u(\tau)\| \leqq \int_{\tau}^{t}\|A u(s)\| d s \leqq \int_{\tau}^{t}\|A a\| d s=|t-\tau|\|A a\|
$$

and $\lim _{t \uparrow T} u(t)=v_{0}$ for some $v_{0} \in X$. By applying the local existence lemma with initial time $T$ and $u(T)=v_{0}$, we obtain a continuation of the solution beyond $T$ contradicting the definition of $T$.

The uniqueness of the solution follows from standard methods of Kato [5] which use the accretiveness of $A$.

REMARK. If we set $u(t)=T(t) a$ we obtain a semigroup of nonlinear operators $\{T(t): t \geqq 0\}$ which map $X$ into $X$. We can say that $\{T(t)\}$ is the semigroup associated with $-A$.

The next theorem yields the $m$-accretiveness of $A$ and provides an exponential representation for $A$.

THEOREM 2.2. If $A$ is a weakly continuous nonlinear accretive operator defined on a reflexive Banach space then $A$ is $m$-accretive, i.e. $R(I+\lambda A)=X$ for all $\lambda \geqq 0$.

Proof. Oharu [8] has shown that if $R\left(I+\lambda_{0} A\right)=X$ for some $\lambda_{0}>0$, then $R(I+\lambda A)=X$ for all $\lambda \geqq 0$. Thus it is sufficient to establish that for all $b_{0} \in X$ there is an $x_{0} \in X$ so that $A x_{0}+x_{0}=b_{0}$. We introduce an operator $A_{1}$ defined by the equation $A_{1} x=A x+x-b$. Clearly $A_{1}$ is also weakly continuous, accretive and everywhere defined. Denote $\left\{T_{1}(t): t>0\right\}$ to be the semigroup associated with $-A_{1}$. Let $f \in F\left(T_{1}(t) x-T_{1}(t) y\right)$. Then

$$
\begin{aligned}
(d / d t)\left(\left\|T_{1}(t) x-T_{1}(t) y\right\|^{2}\right) & =-2 \operatorname{Re}\left\langle(A+I) T_{1}(t) x-(A+I) T_{1}(t) y, f\right\rangle \\
& \leqq-2\left\|T_{1}(t) x-T_{1}(t) y\right\|^{2}
\end{aligned}
$$


and hence

$$
(d / d t)\left(e^{2 t}\left\|T_{1}(t) x-T_{1}(t) y\right\|^{2}\right) \leqq 0
$$

Integrating (2.4) on $(0, t)$ we obtain $\left\|T_{1}(t) x-T_{1}(t) y\right\| \leqq e^{-t}\|x-y\|$.

If $t_{0}>0$, the Banach fixed point theorem guarantees a unique fixed point of the operator $T_{1}\left(t_{0}\right)$. To see that $T_{1}(t) x_{0}=x_{0}$ for all $t \geqq 0$ we merely observe that $T_{1}(t) x_{0}=T_{1}(t) T_{1}\left(t_{0}\right) x_{0}=T_{1}\left(t_{0}\right) T_{1}(t) x_{0}$. Thus,

$$
0=d T_{1}(t) x_{0} /\left.d t\right|_{t=0}=-A_{1} x_{0}
$$

and hence $A x_{0}+x_{0}=b_{0}$.

REMARK. The $m$-accretiveness of $A$ produces an exponential representation for $T(t)$. A recent theorem of Crandall and Liggett [1] yields

$$
T(t) x=\lim _{n \rightarrow \infty}(I+(t / n) A)^{-n} x .
$$

3. Properties of $T(t)$. In this section we develop some properties of semigroups having weakly continuous infinitesimal generators.

DEFINITION 3.1. If $T(t)$ is a semigroup of nonlinear operators, the infinitesimal generator $A$ is the operator defined by the equation $A x=\lim _{h \rightarrow 0}(T(h) x-x) / h$ whenever this limit exists.

It is known that if $A$ is the infinitesimal generator of a semigroup of nonlinear nonexpansive operators $T(t)$, then $-A$ is an accretive operator. If $A$ is everywhere defined, $T(t)$ provides a solution to the Cauchy problem $d u(t) / d t-A u(t)=0, u(0)=x$ and $T(t) x=\lim _{n \rightarrow \infty}(I-(t / n) A)^{-n} x$.

We shall need the following property of weakly continuous accretive operators.

Lemma 3.1. Let $X$ be a reflexive Banach space and $A$ a weakly continuous accretive operator with $D(A)=X$. If $\lambda$ is any positive number then $J_{\lambda}=(I+\lambda A)^{-1}$ is weakly continuous.

Proof. Suppose that $x_{n} \rightarrow x_{0}$, we wish to show that $J_{\lambda} x_{n} \rightarrow J_{\lambda} x_{0}$. Since $A$ is $m$-accretive there exist $\left\{y_{n}\right\} \subset X$ and $y_{0} \in X$ such that $x_{n}=y_{n}+$ $\lambda A y_{n}$ and $x_{0}=y_{0}+\lambda A y_{0}$; hence $J_{\lambda} x_{n}=y_{n}$ and $J_{\lambda} x_{0}=y_{0}$. The weak convergence of $\left\{x_{n}\right\}$ guarantees its boundedness; to see that the sequence $\left\{y_{n}\right\}$ is bounded we utilize the accretiveness of $A$ to observe that $\left\|y_{n}+\lambda A y_{n}\right\|+\|\lambda A 0\| \geqq\left\|y_{n}+\lambda A y_{n}-\lambda A 0\right\| \geqq\left\|y_{n}\right\|$.

Since $\left\{y_{n}\right\}$ is bounded, it contains weakly convergent subsequences. We wish to show that the only weak limit point of $\left\{y_{n}\right\}$ is $y_{0}$. Suppose $\left\{y_{n}^{\prime}\right\}$ is a subsequence which converges weakly to $y_{1}$. Then by the weak continuity of $A$ we have $x_{n^{\prime}}=y_{n^{\prime}}+\lambda A y_{n} \rightarrow y_{1}+\lambda A y_{1}$. However $\left\{x_{n^{\prime}}\right\} \subseteq\left\{x_{n}\right\}$ and hence $y_{n^{\prime}}+\lambda A y_{n^{\prime}} \rightarrow y_{0}+\lambda A y_{0}$. Therefore, $y_{0}+\lambda A y_{0}=y_{1}+\lambda A y_{1}$ and 
hence by the accretiveness of $A$ we have

$$
0=\left\|y_{1}+\lambda A y_{1}-\left(y_{0}+\lambda A y_{0}\right)\right\| \geqq\left\|y_{0}-y_{1}\right\| .
$$

Thus $y_{1}=y_{0}$.

By virtue of the above lemma we ascertain that for any positive integer $n$ the operator $(I+(t / n) A)^{-n}$ maps weakly convergent sequences to weakly convergent sequences. This observation is crucial to the following theorem.

THEOREM 3.1. Let $X$ be a reflexive Banach space and suppose that $T(t)$ is a semigroup of nonlinear contraction operators defined on $X$. If $T(t)$ has an everywhere defined weakly continuous infinitesimal generator $A$ then for each $t_{0} \geqq 0$ the operator $T\left(t_{0}\right)$ is weakly continuous.

Proof. Let $y \in X$ and then $T(t) y$ provides the unique strong solution to the Cauchy problem $u^{\prime}(t)-A u(t)=0, u(0)=y$ and $t \in[0, T)$. Moreover, $-A$ is accretive and hence $T(t) y=\lim _{n \rightarrow \infty}(I-(t / n) A)^{-n} y$. By use of the estimates of Crandall and Liggett [1] we see that

$$
\left\|T(t) y-(I-(t / n) A)_{y}^{-n}\right\| \leqq 2 T n^{-1 / 2}\|A y\| \text { for } t \in[0, T) .
$$

Let $x_{n} \rightarrow x$. Since $A x_{n} \rightarrow A x$, we can choose $M>0$ so that $\|A x\| \leqq$ $\sup \left\|A x_{n}\right\| \leqq M$. Thus if $T>t_{0}$

$$
\begin{aligned}
\left\|T\left(t_{0}\right) x-\left(I-\left(t_{0} / m\right) A\right)^{-n} x\right\| & \leqq 2 T m^{-1 / 2} M \\
\left\|T\left(t_{0}\right) x_{n}-\left(I-\left(t_{0} / m\right) A\right)^{-m} x_{n}\right\| & \leqq 2 T m^{-1 / 2} M .
\end{aligned}
$$

We are now in a position to complete our proof. Let $f \in X^{*}$ and choose $m$ so that

$$
\begin{aligned}
\left|\left(T\left(t_{0}\right) x-\left(I-\left(t_{0} / m\right) A\right)^{-m} x, f\right)\right| & <\varepsilon / 3 \\
\left|\left(T\left(t_{0}\right) x_{n}-\left(I-\left(t_{0} / m\right) A\right)^{-m} x_{n}, f\right)\right| & <\varepsilon / 3 .
\end{aligned}
$$

Since $\left(I-\left(t_{0} / m\right) A\right)^{-m}$ is continuous from the weak topology of $X$ to the weak topology of $X$, we can choose $N$ so that $n \geqq N$ implies that

$$
\left|\left(\left(I-\left(t_{0} / m\right) A\right)^{-m} x-\left(I-\left(t_{0} / m\right) A\right)^{-m} x_{n}, f\right)\right|<\varepsilon / 3 .
$$

Hence we can choose $N$ so that $n \geqq N$ implies $\left|\left(T\left(t_{0}\right) x-T\left(t_{0}\right) x_{n}, f\right)\right|<\varepsilon$.

The next three propositions apply our results to the rest point theory developed by C. Yen [10].

Definition 3.2. Let $\{T(t): t \geqq 0\}$ be a semigroup. Then a point $x_{0}$ is said to be a rest point of $T(t)$ iff $T(t) x_{0}=x_{0}$ for all positive $t$.

Definition 3.3. Let $\{T(t): t \geqq 0\}$ be a semigroup. The set $O_{x}=\{T(t) x$ : $t \geqq 0\}$ is called the orbit of $x$. If $O_{x}$ is bounded for some $x$ we say that $T(t)$ has bounded orbit. 
Proposition 3.1. Let $X$ be a reflexive Banach space and suppose that $\{T(t): t>0\}$ is a semigroup of contractions having an everywhere defined weakly continuous infinitesimal generator. If $T(t)$ has bounded orbit then $T(t)$ has a rest point.

Proposition 3.9 is an immediate consequence of Theorem 3.1 and C. Yen, Theorem 2.22, op. cit.

Definition 3.4. An operator $A$ is said to be coercive if for any bounded subset $M$ of $R(A), \bigcup\left\{A^{-1} x, x \in M\right\}$ is bounded, i.e. $A^{-1}$ maps bounded sets into bounded sets.

We have the following surjectivity result:

Proposition 3.2. If $X$ is a reflexive Banach space and $A$ is a coercive weakly continuous accretive operator with $D(A)=A$, then $R(A)=X$.

Proof. By Theorem 2.2, $A$ is $m$-accretive. Hence Theorem 1.16 of C. Yen, op. cit., applies.

Proposition 3.3. Let $X$ be a reflexive Banach space and suppose that $\{T(t): t>0\}$ is a semigroup of contraction operators having an everywhere defined coercive, weakly continuous infinitesimal generator; then $\{T(t): t \geqq 0\}$ has a rest point.

Proof. Theorem 2.2 and Proposition 3.2 guarantee that there is an $x_{0}$ such that $-A x_{0}=0$. Thus exponential representation

$$
T(t) x_{0}=\lim _{n \rightarrow \infty}(I-(t / n) A)^{-n} x_{0}=x_{0}
$$

demonstrates that $x_{0}$ is a rest point of $\{T(t)\}$.

\section{REFERENCES}

1. M. Crandall and T. Liggett, Generation of semigroups of nonlinear transformations on general Banach spaces, Amer. J. Math. 93 (1971), 265-298. MR 44 \#4563.

2. S. Chow and J. D. Schuur, An existence theorem for ordinary differential equations in Banach spaces, Bull. Amer. Math. Soc. 77 (1971), 1018-1020. MR 44 \#4334.

3. E. Hille and R. S. Phillips, Functional analysis and semi-groups, rev. ed., Amer. Math. Soc. Colloq. Publ., vol. 31, Amer. Math. Soc., Providence, R.I., 1957. MR 19, 664.

4. T. Kato, Accretive operators and nonlinear evolution equations in Banach spaces, Proc. Sympos. Pure Math., vol. 18, Part 1, Amer. Math. Soc., Providence, R.I., 1970, pp. 138-161. MR 42 \#6663.

5. - Nonlinear semigroups and evolution equations, J. Math. Soc. Japan 19 (1967), 508-520. MR 37 \#1820.

6. R. H. Martin, A global existence theorem for autonomous differential equations in a Banach space, Proc. Amer. Math. Soc. 26 (1970), 307-314. MR 41 \#8791. 
7. J. W. Neuberger, An exponential formula for one-parameter semigroups of nonlinear transformations, J. Math. Soc. Japan 18 (1966), 154-157. MR 34 \#622.

8. S. Ôharu, Note on the representation of semigroups on nonlinear operators, Proc. Japan Acad. 42 (1966), 1149-1154. MR 36 \#3167.

9. G. F. Webb, Continuous nonlinear perturbations of linear accretive operators in Banach spaces, J. Functional Analysis 10 (1972), 191-203.

10. C. Yen, The convergence, periodicity and rest point behaviour of orbits in nonlinear semigroups of contractions, Pacific J. Math. (to appear).

Department of Mathematics, University of Houston, Houston, Texas 77004 